\title{
Caterpillar solutions in coupled pendula
}

\author{
MARK LEVI \\ Department of Mathematics, Boston University, 111 Cummington Street, \\ Boston, MA 02215, USA
}

Abstract. The single pendulum is one of the fundamental model problems in the theory of dynamical systems; coupled pendula, or equivalently, two elastically coupled particles in a periodic potential on a line, are a natural extension of intrinsic interest. The system arises in various physical applications and it inherits some rudiments of the behaviour exhibited by its finite-dimensional parent, the sineGordon equation. Among these phenomena are the so-called caterpillar solutions, whose behaviour is reminiscent of solitons. These solutions turn out to have a transparent geometrical explanation. There is an interesting bifurcation picture associated with the system: the parameter region is broken up into the set of 'pyramids' parametrized by pairs of integers; these integers characterize the behaviour of the associated solutions.

\section{Introduction}

In this paper we use geometrical methods of dynamical systems to carry out a detailed analysis of the system of two coupled pendula with damping and forcing:

$$
\begin{aligned}
& \ddot{\phi}_{1}+\gamma \dot{\phi}_{1}+\sin \phi_{1}+k\left(\phi_{1}-\phi_{2}\right)=I_{1}, \\
& \ddot{\phi}_{2}+\gamma \dot{\phi}_{2}+\sin \phi_{2}+k\left(\phi_{2}-\phi_{1}\right)=I_{2} .
\end{aligned}
$$

Results of our analysis of the special case were announced in [10]; one is referred to that note for the details on the motivation and the background. Equation (1.1) is of basic interest; besides having a simple mechanical interpretation as a pair of pendula subject to elastic and torsional coupling (figure 1.1), it serves as a model

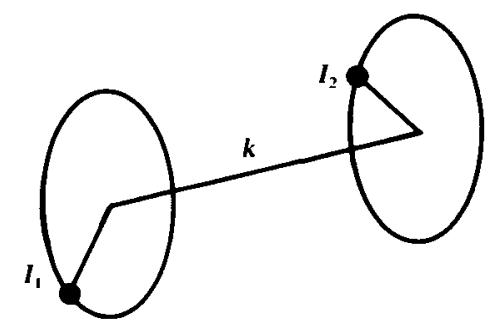

FIGURE 1.1. Pendula on rods are constrained to two parallel vertical planes; the horizontal axis perpendicular to these planes provides torsional coupling. External torques $I_{1}$ and $I_{2}$ are applied to the pendula.

for coupled Josephson junctions [7] as well as for some aspects of charge density waves in anisotropic crystals [6]. Mathematically, equation (1.1) can be obtained as the two-point discretization (in space) of the damped sine-Gordon equation on the finite interval with Neumann boundary conditions. Further details and references are given in [10]. 


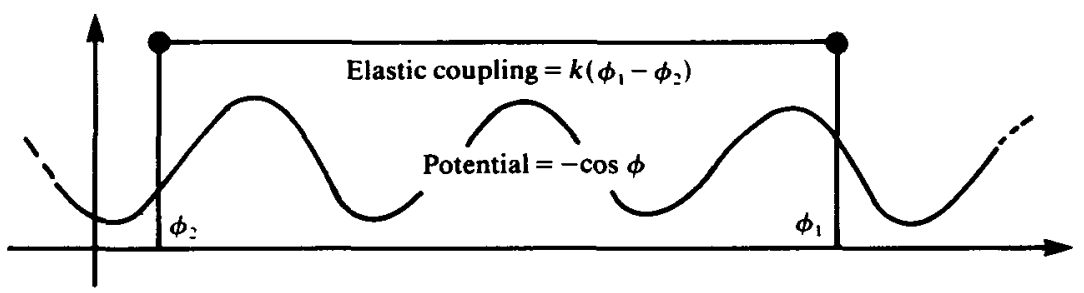

FIGURE 1.2. $\phi_{1}$ and $\phi_{2}$ are the positions of two particles in the periodic potential - $\cos \phi$; the particles are coupled by an elastic 'rubber band' with tension $k\left(\phi_{1}-\phi_{2}\right)$ proportional to the distance, and the particles are subject to constant forces $I_{1}, I_{2}$.

Despite the basic nature of equation (1.1), until recently very little was known about its behaviour. Perhaps the most intriguing and interesting property of the system was discovered by Imry and Schulman [7] numerically and by Zimmerman and Sullivan [24] experimentally. There exist solutions for $k$ not too large, called the 'beating modes' by Imry and Schulman, in which only one pendulum rotates at a time. Another mechanical interpretation of these solutions is shown in figure 1.2. For a proper choice of $k \ll 1, I_{2}=0$ and $I_{1}$ the following motion is observed: as one particle stays in a bounded (by, say, 1) neighbourhood of a minimum of the potential, the other travels to the right, eventually settling in a local well, at which point the previously resting particle climbs out of the well and travels to the right, finally settling in another well, etc. This periodic motion invites the name 'caterpillar' solutions, which we use instead of the term 'beating modes', since the standard beating modes have a simple local and, in fact, linear nature, while the mechanism of the solutions discussed here is non-linear and non-local. Actually, these solutions are the finite-dimensional analogues of solitary waves. This low-dimensional setting allows us to give a simple geometrical explanation of the mechanism of these finite-dimensional 'solitons'.

Equation (1.1) is remarkable in that it is complex enough to exhibit interesting wave-like (non-linear) behaviour inherited from its sine-Gordon parent $\dagger$ and yet simple enough to be amenable to geometric analysis.

\section{Results}

We will give a bifurcation diagram in figure 2.3 , providing a near-complete picture of the behaviour of equation (1.1) for all $k$ less than a certain value $k_{0}=k_{0}(\gamma)$. To help the reader follow the path of least time, we recommend going directly to figure 2.3 and to the main theorem immediately following it, referring to the definitions below as necessary.

\subsection{Definitions of caterpillar solutions}

Definition 1. We will call a solution $\left(\phi_{1}, \phi_{2}\right)$ of equation (1.1) a running periodic solution if there exists (a period) $T>0$ and an integer $m>0$ such that

$$
\phi_{1,2}(T)=\phi_{1,2}(0)+2 \pi m, \quad \dot{\phi}_{1,2}(T)=\dot{\phi}_{1,2}(0) \text {. }
$$

† With damping and forcing, which destroy all the integrals. Very little is known about the qualitative behaviour of that system. Some very interesting results in that direction were obtained in [3]. 


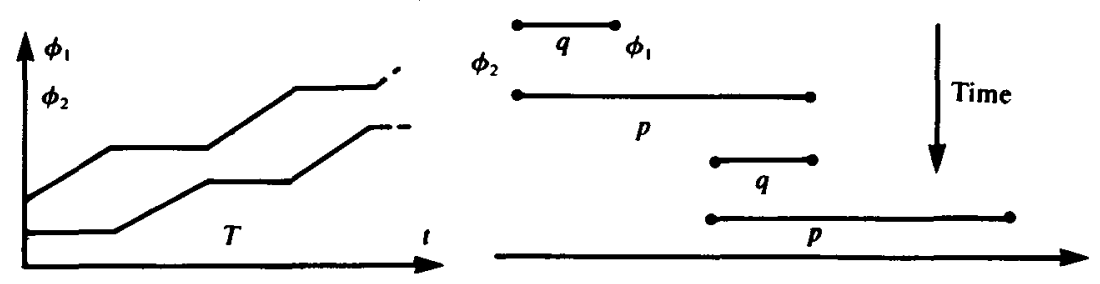

FIGURE 2.1. A 'caterpillar' solution.

Definition 2. A 'caterpillar' solution is a running periodic solution of (1.1) whose period consists of two intervals, during the first of which $\phi_{1}$ increases by $2 \pi m+R_{1}$, $m$ being an integer and $0<R_{1}<\pi / 2$, while $\phi_{2}$ changes by less than $\pi / 2$, and during the second of which $\phi_{1}$ and $\phi_{2}$ exchange roles: $\phi_{2}$ increases by $2 \pi m+R_{2}, 0<R_{2}<$ $\pi / 2$, while $\phi_{1}$ changes by less than $\pi / 2$ (figure 2.1 ).

More precisely, there exists $\tau \in(0, T)$ and an integer such that

$$
\begin{gathered}
\phi_{1}(\tau)-\phi_{1}(0)=2 \pi m+R_{1}, \quad 0<R_{1}<\pi / 2, \\
\left|\phi_{2}(t)-\phi_{2}(0)\right|<\pi / 2 \quad \text { for all } 0 \leq t \leq \tau
\end{gathered}
$$

and

$$
\begin{array}{cc}
\phi_{2}(T)-\phi_{2}(\tau)=2 \pi m+R_{2}, & 0<R_{2}<\pi / 2, \\
\left|\phi_{1}(t)-\phi_{1}(\tau)\right|<\pi / 2, & \tau \leq t \leq T .
\end{array}
$$

Definition 3. A caterpillar solution has the type $(p, q)$ with $p, q \in \mathbf{Z}$ if the distance $\phi_{1}-\phi_{2}$ changes from the minimum $2 \pi q+R_{3}$ to the maximum $2 \pi p+R_{4},\left|R_{3,4}\right|<\pi / 2$ during one period (figure 2.1). Thus $q$ is the smallest and $p$ is the largest integer length of the caterpillar.

To state our main result, i.e. to describe the bifurcation diagram, we need an auxiliary construction which is reproduced here for completeness.

2.2. An auxiliary construction. The key to the construction of the bifurcation diagram of equation (1.1) is the autonomous system

$$
\ddot{\phi}+\gamma \dot{\phi}+\sin \phi+k \phi=a, \quad a=\text { const, }
$$

which governs the motion of one pendulum in our system when the other is held fixed. If the pendulum is wound backwards so that the net torque $a-k \phi$ builds up to, say, 1 , and then released, the pendulum will tumble $O(1 / k)(k \ll 1)$ times before settling in a sink - or a saddle if the initial data lie on the stable manifold. The position of the final sink is very insensitive to the initial condition for the initial conditions 'not too close' to the stable manifold.

Definition 4. The distinguished $\operatorname{sink}(S, 0) \equiv(S(a ; k), 0)$ of equation $(2.1)$ is that sink which captures the solution with initial data

$$
a-k \phi_{0}=1, \quad \dot{\phi}_{0}=0 .
$$

The first saddle equilibrium to the right of $S(a ; k)$ is denoted $S a(a ; k)$; we will call it the distinguished saddle. When $\left(\phi_{0}, \dot{\phi}_{0}\right)$ lies on the stable manifold of a saddle, the distinguished $\operatorname{sink} S(a ; k)$ is undefined; for such $(a, k)$ the function $S$ 


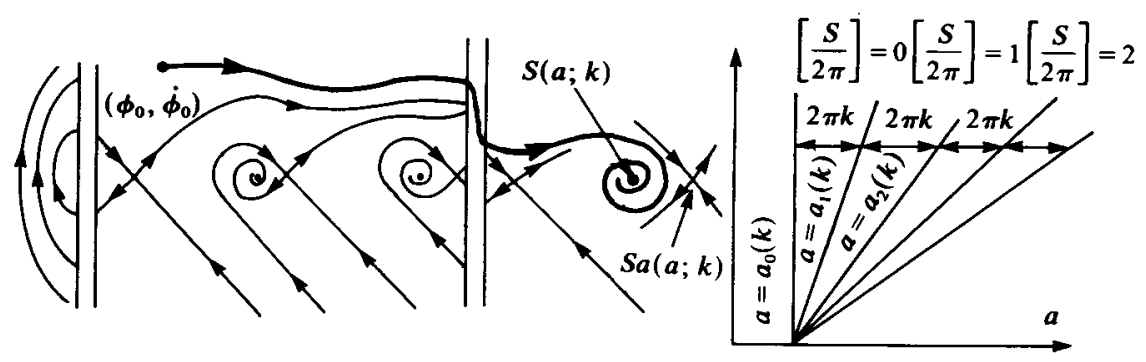

FIGURE. 2.2. Phase portrait of equation (2.1) and the discontinuity lines $a=a_{m}(k)$ of $S(a ; k)$.

undergoes a jump (by $2 \pi+O(k)$ ). We list here the properties of $S(a ; k)$ needed below.

(1) Periodicity: $S(a+2 \pi k ; k)=S(a ; k)+2 \pi$.

(2) $(\partial / \partial a) S(a ; k)=1 /(\cos S+k) \geq c(\gamma)>0$, where $c(\gamma)$ depends on $\gamma$ only.

(3) $k S(a ; k)=a-T_{\mathrm{c}}+\rho,|\rho|<3 \pi k$, where $T_{\mathrm{c}}=T_{\mathrm{c}}(\gamma)$ is that (unique) value of torque $T$ which gives rise to the saddle-saddle heteroclinic connection for the single damped pendulum system $\ddot{\phi}+\gamma \dot{\phi}+\sin \phi=T$. (See [22], [1] and [11] for the details on this system.)

(4) The discontinuities of $S(a ; k)$ form a countable set of curves $a=a_{m}(k), m \in \mathbf{Z}$ in the $(a, k)$-plane (figure 2.2). These curves fan out of the point $\left(T_{c}, 0\right)$, as follows from:

(5) The jump in $S$ across each curve (crossing to the right) is $2 \pi+O(k)$. Finally, $a_{m+1}(k)=a_{m}(k)+2 \pi k$.

Proof of (1)-(5) amounts to the phase plane analysis of equation (2.1) and is omitted.

2.3. Construction of the caterpillar domains $C_{p, q}$. Consider the half-planes of discontinuity of two functions $S\left(I_{1}+k \pi / 2 ; k\right)$ and $S\left(I_{2}+k \pi / 2 ; k\right)$, both defined in the $\left(I_{1}, I_{2}, k\right)$-half-space $k>0$. Each wedge $a_{m}(k)<I_{2}+k \pi / 2<a_{m+1}(k)$ is assigned its own integer

$$
q=-\left[\frac{1}{2 \pi} S\left(I_{2}+\frac{k \pi}{2} ; k\right)\right] ; \dagger
$$

similarly, the wedges $a_{m}(k)<I_{1}+k \pi / 2<a_{m+1}(k)$ which are parallel to the $I_{2}$-axis are enumerated by

(figure 2.3).

$$
p=\left[\frac{1}{2 \pi} S\left(I_{1}+\frac{k \pi}{2} ; k\right)\right]
$$

The discontinuity half-planes $I_{1}+k \pi / 2=a_{m}(k), I_{2}+k \pi / 2=a_{m}(k), k>0$ subdivide the half-space $k>0$ into pyramidal cells; each cell is labelled by a pair $(p, q)$ of integers indicating the numbers of wedges to which the cell belongs. We define the caterpillar domain $C_{p, q}$ by removing an $\alpha k$-neighbourhood of the boundary from each pyramid, where $\alpha$ can be chosen arbitrarily small; we pick $\alpha=\frac{1}{100}$ to avoid technicalities.

† With [ ] denoting the integer part. 


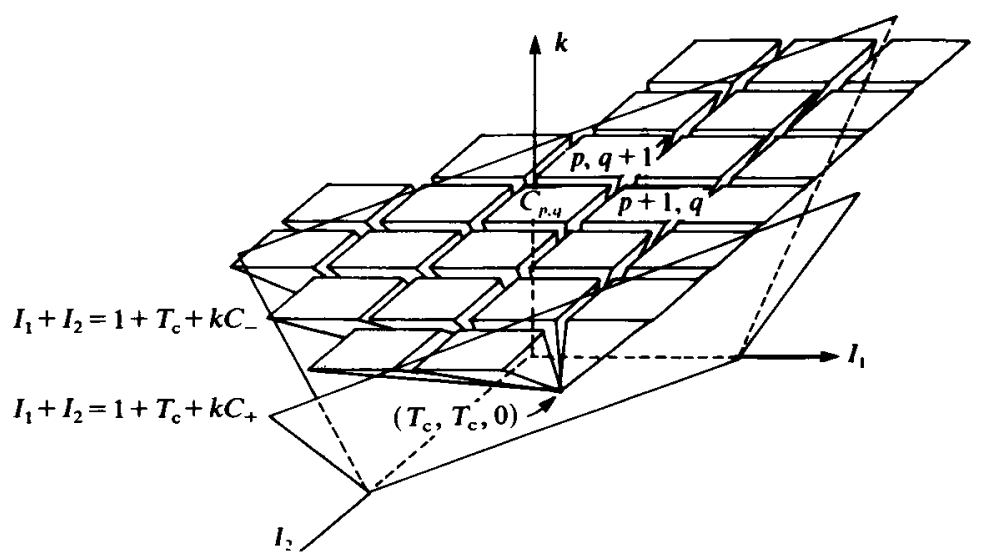

Figure 2.3. Caterpillar domains $C_{p, q}$ for equation (1.1) are the pyramids $C_{p, q}$ with the common vertex at $\left(T_{\mathrm{c}}, T_{\mathrm{c}}, 0\right)$.

MAIN THEOREM. Fix $\gamma>0$ and fix two arbitrary constants $C_{+}>C_{-}>0$. $\dagger$ There exists $k_{0}=k_{0}\left(\gamma, C_{-}, C_{+}\right)>0$ such that for any parameter point $\left(I_{1}, I_{2}, k\right)$ satisfying $0<k<k_{0}$ and lying in the intersection of a caterpillar domain $C_{p, q}$ with the wedge $\ddagger$ $1+T_{\mathrm{c}}+k C_{-}<I_{1}+I_{2}<1+T_{c}+k C_{+}$, figure 2.3, equation (1.1) possesses a pair of $(p, q)$-caterpillar solutions, one exponentially asymptotically stable and the other unstable. The orbits of these two solutions are within distance $\pi$ of each other.

COROLlaRY 1. Letting $I_{2}=0, I_{1} \equiv I$ we obtain the bifurcation diagram in the $(I, k)$ plane by slicing the diagram in figure 2.3 by the plane $I_{2}=0$ (figure 2.4).

We note that an alternative way to obtain this diagram is to take the square lattice in a $k=$ const plane, defined by the lines $I_{1}=a_{m}(k), I_{2}=a_{m}(k), m \in \mathbf{Z}$, and to project it centrally onto the $I_{2}=0$ plane from the point $\left(T_{c}, T_{c}, 0\right)$. In other words, the lines

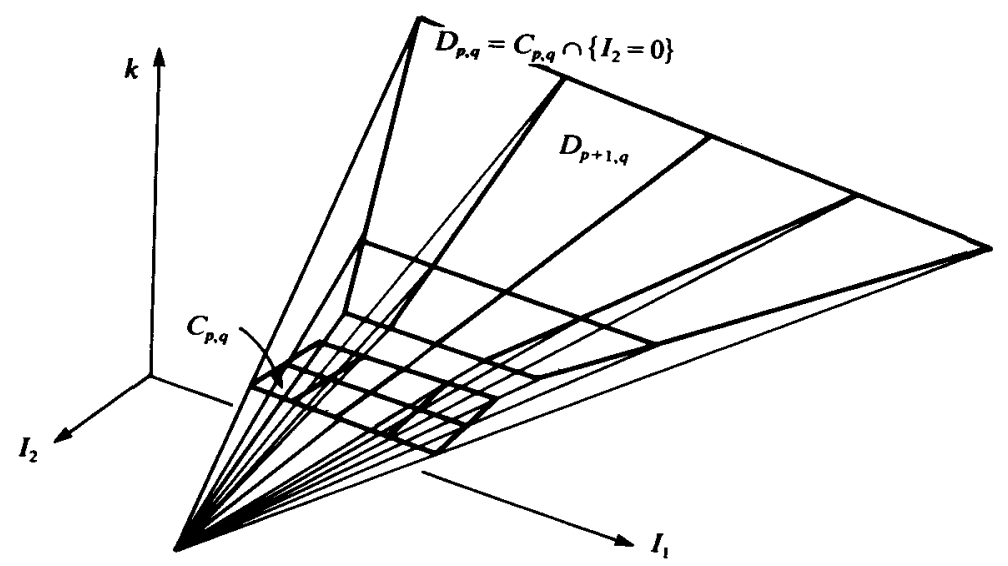

FIGURE 2.4. Bifurcation diagram for the system (1.1) with $I_{1}=I, I_{2}=0 . D_{p, q}$ are the intersections of the caterpillar domains $C_{p, q}$ with the $I_{2}=0$ plane.

$\ddagger$ We recall that $T_{c}=T_{c}(\gamma)$ was defined as the heteroclinic value of $T$ in the equation $\ddot{\phi}+\gamma \dot{\phi}+\sin \phi=T$. 


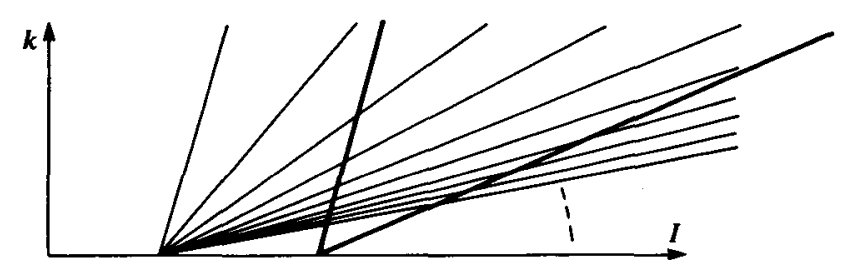

FIGURE 2.5. Bifurcation diagram for $I_{1}=I_{2}=I$.

defining the lattice in the $I_{2}=0$ plane are the shadows of the lines defining the rectangular lattice when the source of light is placed at $\left(T_{\mathrm{c}}, T_{\mathrm{c}}, 0\right)$.

Corollary 2. Setting $I_{1}=I_{2}=I$ gives rise to the system with 'constant bias'. In this case $p=-q$; in the resulting motion each particle alternately overtakes the other by $2 \pi p+E,|E|<\pi / 2$. The bifurcation diagram looks somewhat simpler (figure 2.5).

In this particular case of $I_{1}=I_{2}$ one can interpret (1.1) as describing two particles in the 'staircase' potential $-I \phi-\cos \phi$. The caterpillar motion looks like that of a 'slinky'.

Fixing $C_{+} \gg C_{-}>0$ and fixing $k<k_{0}\left(C_{-}, C_{+}, \gamma\right)$, we vary the slope $I$ through the long interval $\frac{1}{2}\left(1+T_{\mathrm{c}}+k C_{-}\right)<I<\frac{1}{2}\left(1+T_{\mathrm{c}}+k C_{+}\right)$. While doing so, we will pass 'forbidden' intervals of small length $<\alpha k$ for which no caterpillar solutions exist; in the remaining intervals of length $2 \pi k-\alpha k$ we will obtain $(p,-p)$-caterpillars corresponding to the particles overtaking one another alternately with maximal separation reaching $2 \pi p+R,|R|<\pi / 2$. Here

$$
p=\left[\frac{1}{2 \pi} S\left(I+\frac{k \pi}{2} ; k\right)\right] .
$$

'Kemark 1. Global behaviour. One can actually give a complete analysis of the system by specifying all possible fates of its solutions. As it turns out, any solution does one of the following: it either (1) tends to one of the two caterpillars, (2) tends to one of the $O(1 / k)$ equilibria or (3) enters a tubular neighbourhood of the synchronous solution, i.e. the solution for which the pendula undergo an in-phase rotation with the phase difference $\phi_{1}-\phi_{2}$ bounded by a constant independent of $k+$

The incompleteness of this description is due to the lack of full understanding of the flow in the tubular neighbourhood, although some interesting examples of coexisting solutions are known [7]. It is unclear, in particular, whether every solution tends to a periodic one.

Remark 2. The so-called 'period-adding' phenomenon in forced oscillators [4] bears a certain similarity to the bifurcation leading to the change of integers $p, q$. In fact, while the effect of period-adding in forced oscillators is different from the effect of change of relaxation integer $p-q$, there is a strong similarity, both in the underlying geometry (despite the difference of dimensions between the problems) and in the additive nature of the change.

† We omit the proof of the existence of synchronous solutions. One can give such a proof using the Bohl-Brower fixed point theorem just as was done in [11] in proving the existence of periodic solutions for the damped sine-Gordon equation. 
Remark 3. Relaxation oscillations. It is interesting to observe that each pendulum taken individually behaves as a relaxation oscillator: the slow ('standing') phase alternates with the fast ('running') phase. It is remarkable that this relaxation behaviour is unexpected $a$ priori, unlike in the standard cases of fast-slow systems in the plane, where the solutions 'fall' and 'drift' alternately [20]. The full solution $\Phi=\left(\phi_{1}, \dot{\phi}_{1}, \phi_{2}, \dot{\phi}_{2}\right)$ in $\mathbf{R}^{4}$, rather than its two components, does not have the same fast-slow behaviour.

Remark 4. Taking small $k$ amounts to dealing with a singular perturbation problem. Taking $k \rightarrow 0$ leads to a singular limit: the period as well as the separation between $\phi_{1}$ and $\phi_{2}$ tend to $\infty$. If we set $k=0$, all information on the interaction is lost, so that starting the perturbations procedure with $k=0$ is of no help.

Remark 5. Invariance of the bifurcation diagram. Linear transformation $T:\left(I_{1}, I_{2}, k\right) \mapsto\left(I_{1}+2 \pi k, I_{2}-2 \pi k, k\right)$ in the parameter space leaves the bifurcation diagram invariant and is equivalent to the shift in the domain indices

$$
T C_{p, q}=C_{p+1, q+1} \text {. }
$$

Proof. Let $\left(I_{1}, I_{2}, k\right) \in C_{p, q}$ and let $\left(\phi_{1}, \phi_{2}\right)$ be any solution satisfying equation (1.1). Then $\left(\bar{\phi}_{1}, \bar{\phi}_{2}\right) \equiv\left(\phi_{1}+2 \pi, \phi_{2}\right)$ is a solution to (1.1) with $\bar{I}_{1}=I_{1}+2 \pi k$ replacing $I_{1}$ and $\bar{I}_{2}=I_{2}-2 \pi k$ replacing $I_{2}$. Now let $\left(\phi_{1}, \phi_{2}\right)$ be a $(p, q)$-caterpillar solution of (1.1). Then $\left(\phi_{1}+2 \pi, \phi_{2}\right)$ is a $(p+1, q+1)$-caterpillar solution of the equation with $\bar{I}_{1}, \bar{I}_{2} ;$ thus $\left(\bar{I}_{1}, \bar{I}_{2}, k\right) \in C_{p+1, q+1}$.

Remark 6. The linear nature of damping and coupling plays no role in the proofs, and the theorem could easily be extended to include non-linear dissipation and coupling subject to mild monotonicity conditions. This can be done using the same arguments as the ones we use below; we look at the simplest case to avoid the unnecessary technicalities.

Open questions. (1) What is the behaviour in the tubular neighbourhood of the synchronous solution? This neighbourhood can be given explicitly as the neighbourhood of the curve (not the solution) in $\mathbf{R}^{4}$ given by $\left(\phi_{1}, \dot{\phi}_{1}, \phi_{2}, \dot{\phi}_{2}\right)$ with $\phi_{2}-\phi_{1}=$ $\left(I_{2}-I_{1}\right) / 2 k, \quad \dot{\phi}_{1}=p\left(\phi_{1},\left(I_{1}+I_{2}\right) / 2\right), \quad \dot{\phi}_{2}=p\left(\phi_{2},\left(I_{1}-I_{2}\right) / 2\right)$, where $\dot{\phi}=p(\phi, T, \gamma)$ gives the graph of the running periodic solution of $\ddot{\phi}+\gamma \dot{\phi}+\sin \phi=T$ in the $(\phi, \dot{\phi})$ plane [11].

(2) What is the qualitative behaviour of the chain of $n$ pendula?

(3) What is the effect of periodic forcing $I=I_{0}+\varepsilon \sin t$ ?

\section{Proof of the theorem}

3.1. Heuristic discussion. Heuristic arguments for the particular case $I_{2}=0$ can be found in [10]; here we indicate a brief outline for completeness. The mechanism responsible for the caterpillar effects works as follows.

Assume that one pendulum, say $\phi_{1}$, starts near a (slowly moving) sink of the first equation $\ddot{\phi}_{1}+\gamma \dot{\phi}_{1}+\sin \phi_{1}+k \phi_{1}=k \phi_{2}+I_{1}$ with the right-hand side treated as a near-constant ( $k$ is small). Assume that the other pendulum, $\phi_{2}$, is running meanwhile according to $\ddot{\phi}_{2}+\gamma \dot{\phi}_{2}+\sin \phi_{2}+k \phi_{2}=k \phi_{2}+\left(I_{2}-k \phi_{1}\right)$; the last difference in parentheses changes by $O(k)$ during the time that $\phi_{1}$ stays near its sink. Now, if a 
caterpillar solution is to exist, then $\phi_{1}$ will have to start, i.e. the sink of the first equation which has held $\phi_{1}$ must disappear in a saddle-node bifurcation at about the same time that $\phi_{2}$ stops running, i.e. when the equation for $\phi_{2}$ with the right-hand side treated as a constant acquires a heteroclinic connection. To summarize, a vague and approximate necessary condition for a caterpillar is the near-simultaneous occurrence of the saddle-node bifurcation for one pendulum with the heteroclinic bifurcation for the other.

So far this explains or predicts nothing about caterpillar solutions. The crucial point is this: near the saddle-node the vector field is quadratically slow, while near a saddle it is linearly slow. Consequently, it should take 'much longer' for a point (e.g. $\left.\left(\phi_{1}, \dot{\phi}_{1}\right)\right)$ to leave the saddle-node neighbourhood than it takes a point (e.g. $\left.\left(\phi_{2}, \dot{\phi}_{2}\right)\right)$ to pass by a saddle (if it is not too close to its stable manifold).

In physical terms, the slowness of the vector field near the saddle-node can hold one pendulum slow long enough, providing the other enough time to lose its speed and to settle into an equilibrium. The parameters $I, k$ have to be adjusted so that when one pendulum settles, the torque on the other is larger than 1 (so that it will start) and yet not too large (so that the locking effect of the saddle-node be still valid). This is expressed by equations (3.12) (for $\phi_{1}$ ) and (3.13) (for $\phi_{2}$ ) below. Furthermore, one must avoid parameter values for which the points come too close to a stable manifold of the saddle; this condition is responsible for breaking up the bifurcation diagram into wedges $C_{p, q}$; cf. (3.14) and (3.15).

It is important to point out that the requirement of the near-simultaneous occurrence of the saddle-node and the heteroclinic bifurcations is much less rigid than it may seem. In fact, the smaller $k$ is, the more lax this condition becomes: the locking effect of the saddle-node becomes stronger and stronger. This suggests that the caterpillar phenomenon is quite robust, which is in fact borne out in the analysis below. In particular, the number of caterpillars is arbitrarily large if $k>0$ is small enough; in fact, the aperture $C_{+}-C_{-}$of the dihedral angle in figure 2.3 can be made to include arbitrarily many domains $C_{p, q}$, i.e. the number of distinct caterpillar solutions tends to $\infty$ and $k \rightarrow 0$.

In the next section we present the proof of the theorem for the case $I_{2}=0, I_{1}=I$; the general case is a simple modification.

3.2. An outline of the proof. The proofs are given for the case $I_{2}=0, I_{1} \equiv I$. The general case involves no extra arguments. We will prove the existence of two caterpillar solutions by constructing Poincaré sections in $\mathbf{R}^{4}$. For the stable caterpillar we will construct two three-dimensional sections $\Sigma_{1}$ and $\Sigma_{2}$ transversal to the flow of (2.2) in $\mathbf{R}^{4}$ such that for proper values of $I$ and $k$ every orbit starting in $\Sigma_{1}$ crosses some $\tau$-translate $\dagger$ of $\Sigma_{2}$ and vice versa (figure 3.1). This will define two section maps, $P_{i j}: \Sigma \mapsto \Sigma_{j}(\bmod \tau), i \neq j, i, j=1,2$, whose composition $P=P_{21} \circ P_{12}: \Sigma_{1} \mapsto \tau^{m} \Sigma_{1}$ is into $(\bmod r)$ and thus has a fixed point $\bmod \tau$. More precisely, we will show that for $(I, k)$ as above, $P_{12}: \Sigma_{1} \rightarrow \tau^{p} \Sigma_{2}$ and $P_{21}: \Sigma_{2} \rightarrow \tau^{-q} \Sigma_{1}$ are into, where $p>q>0$ are

$\dagger$ We recall that $\tau$ is the translation in the $\phi_{1}$ - and $\phi_{2}$-direction $\tau\left(\phi_{1}, \psi_{1}, \phi_{2}, \psi_{2}\right) \mapsto\left(\phi_{1}+2 \pi, \psi_{1}, \phi_{2}+2 \pi\right.$, $\left.\psi_{2}\right)$. 


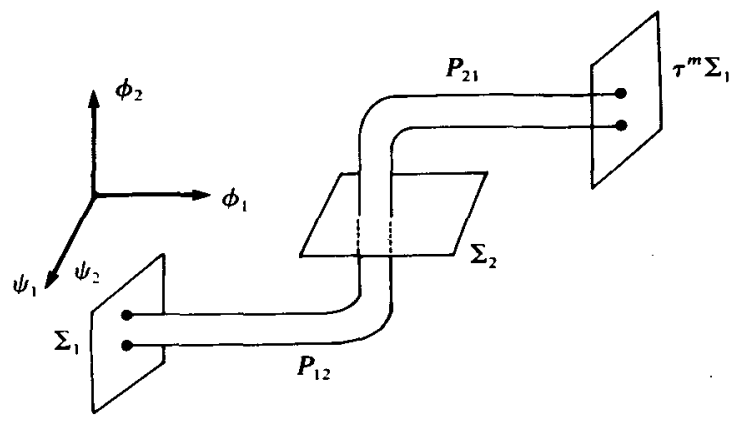

Figure 3.1. Poincaré sections $\Sigma_{1}, \Sigma_{2}$ in $\mathbf{R}^{4}$.

the integers corresponding to the domain $\mathscr{D}_{p, q}$. This shows the existence of a running periodic solution whose homotopy type in the phase cylinder $S^{1} \times R^{3}$ is $m=p-q$. We will prove exponential stability of this solution in $\$ 3.7$.

Existence of the unstable caterpillar is shown in a similar way by constructing different transversal sections $\sigma_{1}$ and $\sigma_{2}$. The composition map $Q: Q_{21} \circ Q_{12}$ deforms the 'cube' $\sigma_{1}$ as shown in figure 3.2; that is, the degree of the map $Q$ relative to $\sigma_{1}$ is -1 , which shows the existence of a fixed point $\bmod \tau$ for the map $Q$. Figure 3.2 also suggests that the point, and thus the corresponding caterpillar, is hyperbolic.

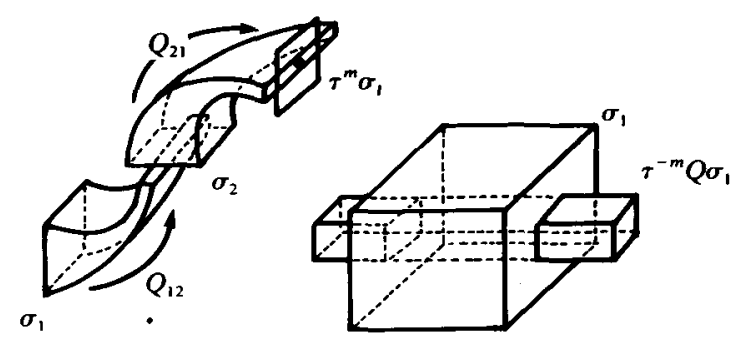

Figure 3.2. Poincaré sections $\sigma_{1}, \sigma_{2}$ giving rise to the unstable caterpillars.

3.3. Construction of Poincaré sections. We first give a formal construction of sections $\Sigma_{1}$ and $\Sigma_{2}$ and follow it up with a short intuitive motivation. Fix $r>0$, to be specified later.

Definition of $\Sigma_{1}$. Let $\Sigma_{1}$ be a direct product of the segment $S_{1}$ in the $\Phi_{1} \equiv$ $\left(\phi_{1}, \psi_{1}\right)$-plane and the box $B_{1}$ in the $\Phi_{2}$-plane (figure 3.3), where $S_{1}$ and $B_{1}$ are defined as follows.
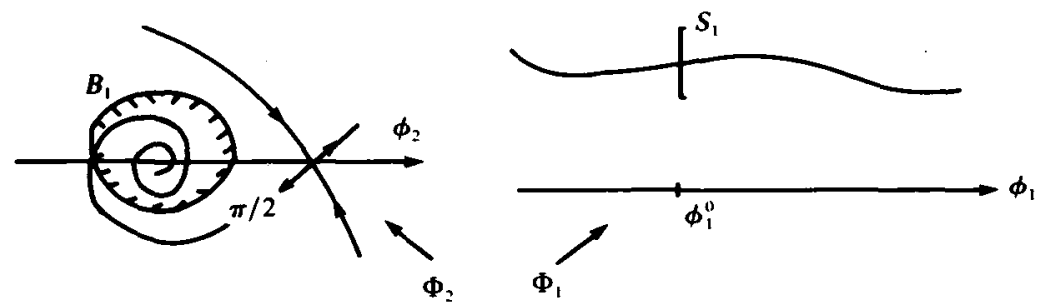

FIGURE 3.3. Projections of the section $\Sigma_{1}$ onto $\phi_{1}$ - and $\phi_{2}$-planes: $\Sigma_{1}=S_{1} \times B_{1}$. 
Segment $S_{1}$. Fix any value of $\phi_{1}^{0}$ (the $\phi_{1}$-coordinate of $S_{1}$ ) which satisfies

$$
T_{\mathrm{c}}+c \leq 1-k \phi_{1}^{0} \leq 1-c, \quad c>0
$$

e.g. let $I-k \phi_{1}^{0}=\left(1+T_{\mathrm{c}}\right) / 2$ and let the vertical coordinates of the segment be given by

$$
\frac{1}{2} p\left(\phi_{1}^{0}, I-k \phi_{1}^{0}\right) \leq \dot{\phi}_{1} \leq 2 p\left(\phi_{1}^{0}-k \phi_{1}^{0}\right) .
$$

Here $\psi=p(\phi, F)$ is the running periodic solution of the single pendulum equation $\ddot{\phi}+\gamma \dot{\phi}+\sin \phi=F$.

Box $B_{1}$. This is defined as the $r$-neighbourhood of the sink nearest $(\pi / 2,0)$ of the autonomous equation

$$
\ddot{\phi}+\gamma \dot{\phi}+\sin \phi+k \phi=k \phi_{1}^{0} .
$$

This completes the definition of $\Sigma_{1}$.

Definition of $\Sigma_{2} . \Sigma_{2}=S_{2} \times B_{2}$, where the segment $S_{2}$ is given by $\left\{\left(\phi_{2}^{0}, \phi_{2}\right)\right\}$ with a fixed $\phi_{2}^{0}$ subject to

$$
T_{\mathrm{c}}+c \leq-k \phi_{2}^{0} \leq 1-c, \quad c>0 ; \quad \text { e.g. }-k \phi_{2}^{0}=\left(1+T_{\mathrm{c}}\right) / 2,
$$

and with $\dot{\phi}_{2}$ subject to

$$
\frac{1}{2} p\left(\phi_{2}^{0},-k \phi_{2}^{0}\right) \leq \dot{\phi}_{2} \leq 2 p\left(\phi_{2}^{0},-k \phi_{2}^{0}\right) .
$$

Box $B_{2}$ is defined as the $r$-neighbourhood of that sink of

which lies near $(\pi / 2,0)$.

$$
\ddot{\phi}+\gamma \dot{\phi}+\sin \phi+k \phi=I+k \phi_{2}^{0}
$$

Sections $\sigma_{1}$ and $\sigma_{2}$. These are defined in the same way except that $B_{1}, B_{2}$ are replaced by the $r$-neighbourhoods $b_{1}, b_{2}$ of the saddles of (3.3) and (3.6) which lie nearest $(\pi / 2,0)$ (figure 3.4).
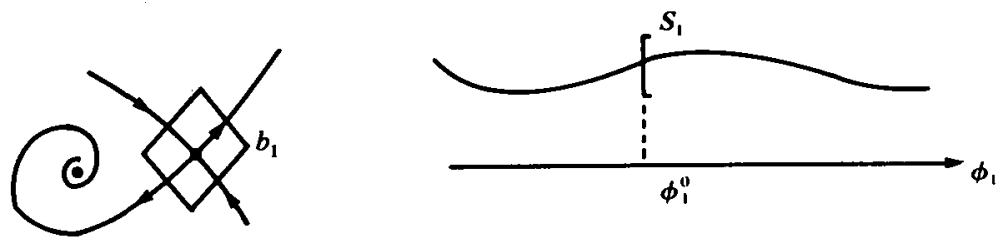

FIGURE 3.4. Section $\sigma_{1}=S_{1} \times b_{1}$.

3.4. Fundamental lemmas: A, B, C. In the following two key lemmas (A and B) we extract the properties of solutions of a class of equations

$$
L \phi \equiv \ddot{\phi}+\gamma \dot{\phi}+\sin \phi+k \phi=b(t)
$$

when these solutions undergo transitions, i.e. when they 'start' or 'stop'. Lemma $A$ will be used to show that the starting is slow (cf. the heuristic argument in $\S 2.3$ ), while Lemma B will apply to showing that the capture into the sink is relatively fast provided $b(t)$ avoids 'bad' values.

The statements of lemmas below use a quantity associated with the autonomous equation

$$
\ddot{\phi}+\gamma \dot{\phi}+\sin \phi+k \phi=a, \quad a=\text { const. }
$$


This quantity is the value of $a$ which gives rise to the saddle-node bifurcation near $\pi / 2$; we denote this value by $a_{\mathrm{sn}}=a_{\mathrm{sn}}(k)$. In other words $a_{\mathrm{sn}}(k)$ is defined by the requirement that the equilibrium equation

$$
\sin \phi+k \phi=a_{\mathrm{sn}}(k)
$$

must have a double root near $\pi / 2$; one concludes from this that

$$
a_{\mathrm{sn}}(k)=1+(\pi / 2) k+O\left(k^{2}\right) .
$$

Lemma A. Assume that for some $c>0, b(t)$ satisfies $\dagger$

$$
\begin{aligned}
& \text { (i) } b(0) \leq a_{\mathrm{sn}}-c, \\
& \text { (ii) }|\dot{b}(t)| \leq c k \quad \forall t \geq 0, \\
& \text { (iii) } b(t) \leq a_{\mathrm{sn}}+C k \quad \forall t \geq 0 .
\end{aligned}
$$

For any $\gamma>0, \beta>0$ there exist $r=r(\gamma), k_{0}=k_{0}(\beta), c=c(\beta)$ and $C$ such that if (iv) $(\phi(0), \dot{\phi}(0)) \in r$-neighbourhood of the sink of $L \phi=b(0)$ which is nearest $\pi / 2$, then for all $k<k_{0}$ we have

$$
|\phi(t)-\pi / 2|<\beta \quad \text { for } t_{\mathrm{sn}} \leq t \leq t_{\mathrm{sn}}+c(\beta) k^{-1 / 2}
$$

and

$$
\phi(t)<\pi / 2+C k \quad \text { for } t \leq t_{\mathrm{sn}},
$$

where $t_{\mathrm{sn}}$ is the smallest $t>0$ for which $b(t)=a_{\mathrm{sn}}$.

The key point of this lemma is that $\phi(t)$ stays near $\pi / 2$ for the time $\sim 1 / \sqrt{k}$ after the saddle-node bifurcation.

Before stating the lemma, we need one notation. Let $\psi=p(\phi, F)$ be the graph in the $(\phi, \dot{\phi})$-plane of the running periodic solution of the forced pendulum equation $\ddot{\phi}+\gamma \dot{\phi}+\sin \phi=F ; p$ is $2 \pi$-periodic in $\phi$ and is defined only for $F \geq T_{\mathrm{c}}$.

LEMMA B. Let $\delta=\delta(k)$ satisfy $e^{-k^{-p}} \leq \delta(k) \leq \pi k$ with some $0<p<1$. Choose $c_{1}, c_{2}, c_{6}>0$ subject to $T_{c}+c_{1}<1-c_{2}, c_{6}>2 \pi$. There exists $r=r\left(c_{1}, c_{2}, \gamma\right)$ such that for any solution $\phi(t)$ of (3.7) with $b(t) \in\left[a_{m}+\delta(k), a_{m+1}-\delta(k)\right]$ for all $t$ and with initial conditions subject to

$$
T_{\mathrm{c}}+c_{1} \leq a_{m}-k \phi(0) \leq 1-c_{2}
$$

and

$$
\left|\dot{\phi}(0)-p\left(\phi(0), a_{m}-k \phi(0)\right)\right| \leq r,
$$

(i) -(iv) below hold for $k$ small enough.

(i) If $t>0$ is large enough so that for some $c_{3}, c_{4}>0$

$$
k \phi(t) \geq k \phi(0)+c_{3}
$$

and

$$
b(t)-k \phi(t) \geq T_{\mathrm{c}}+c_{4},
$$

then for some $c_{5}>0$

$$
\left|\dot{\phi}(t)-p\left(\phi(t), a_{m}-k \phi(t)\right)\right| \leq c_{5} k
$$

$\dagger$ Throughout this paper $c, c_{1}, c_{2}, \ldots$ stand for constants independent of $\gamma, k$. 
Given any $c_{6}>2 \pi$ and $r>0$ there exist $t^{\prime}=t^{\prime}\left(c_{6}, k\right)$ and $t^{\prime \prime}=t^{\prime \prime}(r, k)$ such that (ii), (iii), (iv) hold.

$$
\text { (ii) } \phi\left(t^{\prime}\right)=S a\left(a_{m}, k\right)-c_{6} \text {; }
$$

such $t^{\prime}$ is unique.

(iii) $|(\phi(t), \dot{\phi}(t))-(S(a, k), 0)|<r \quad$ for any $t \geq t^{\prime \prime}, \quad a \in\left(a_{m}, a_{m+1}\right)$.

(iv) $0<t^{\prime \prime}-t^{\prime}<c \ln \frac{1}{\delta(k)}, \quad t^{\prime}>\frac{c}{k}, \quad$ figure 3.5 ,

for some $c=c(r)>0$. Furthermore, fixing any $c>0$, we have for $k$ small enough

$$
\text { (v) } \phi(t) \leq S a(\bar{b} ; k)+c \quad \forall t \geq 0 \text {, }
$$

where $\bar{b}=\max _{t} b(t)$; the condition on $b(t)$ avoiding $a_{m}(k)$ is not necessary for this last statement of the lemma.

We will also need a simple but crucial 'parametric non-resonance' lemma which states roughly that the pendulum (3.7) cannot 'start' as long as the net torque on it is $<1$ and as long as that torque changes not faster than $O(k)$ :

LEMMA C. If $b(t)$ satisfies the following estimates with all $k>0$ small enough:

$$
\begin{aligned}
& b(0) \leq a_{\mathrm{sn}}-c, \\
& |\dot{b}(t)| \leq c k \quad \text { and } \quad b(t) \leq a_{\mathrm{sn}} \quad \text { for } 0 \leq t \leq T, \\
& b(T) \geq a_{\mathrm{sn}}-c k \quad \text { for some } c>0,
\end{aligned}
$$

then any solution of (3.7) with $(\phi, \dot{\phi})_{t=0}$ subject to (iv) in Lemma A satisfies $\phi(t) \leq \pi / 2+c k, \forall t \in(0, T)$.

Proof of lemma A consists of two parts: first we show that for $t \leq t_{\mathrm{sn}}=$ $\inf \left\{t: b(t)=a_{\text {sn }}\right\}$ the solution $\Phi(t)=(\phi, \dot{\phi})$ with $\Phi(0)$ satisfying (iv) with $r>0$ small enough does not cross the strong stable manifold of the saddle-node of the auxiliary autonomous system $L \psi=a_{\mathrm{sn}}$; in the second part of the proof we show that it takes time $\geq c / \sqrt{k}$ to leave a $\beta$-neighbourhood of the saddle-node.

Part 1. For $0<t<t_{\mathrm{sn}}, \Phi(t)$ does not cross the part BCD of the curve $W^{s}$, the strong stable manifold of the saddle-node of the flow of $L \psi=a_{\text {sn }}$ (figure 3.5(a)). Indeed, at a point $(\phi, \psi) \in W^{s}$ the velocity vector $\dot{\Phi}=(\psi,-\sin \phi-k \phi+b(t))$ lies below the tangent vector $V=\left(\psi,-\sin \phi-k \phi+a_{\mathrm{sn}}\right)$ to $W^{\mathrm{s}}$ since $b(t)<a_{\mathrm{sn}}$ for $0<t<t_{\mathrm{sn}}$ (figure 3.5(a)). Finally, $\Phi$ cannot reach the arc $\mathrm{AB}$ of $W^{\mathrm{s}}$ shown in the figure, as follows,

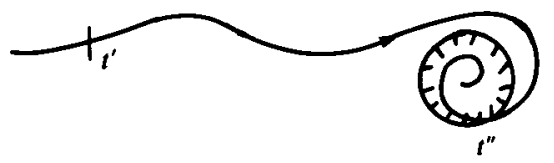

Figure 3.5. Times $t^{\prime}$ and $t^{\prime \prime}$ satisfy $t^{\prime \prime}-t^{\prime} \leq c \ln (1 / k)$.

from assumptions (ii) and (iv) of the lemma, provided $r$ is chosen small enough (independently of $k$ ).

Part 2. To show that it takes time $\geq c k^{-1 / 2}$ for $\Phi(t)$ to leave the $\beta$-neighbourhood of the saddle-node of $L \phi=a_{\mathrm{sn}}$, we bring the non-autonomous vector field of 
$L \phi=b(t)$ to a normal form using a normalizing transformation for the autonomous vector field of $L \phi=a_{\mathrm{sn}}$. In the neighbourhood of the saddle-node $\left(\phi_{\mathrm{sn}}, 0\right)$ the deviations $\bar{\phi}=\phi-\psi_{\mathrm{sn}}$ and $\bar{\psi}=\psi$, where $L \phi=b(t)$, satisfy

$$
\dot{\phi}=\bar{\psi}, \quad \dot{\psi}=-\gamma \bar{\psi}+\frac{1}{2} \bar{\phi}^{2}+b(t)-a_{\mathrm{sn}}+o\left(\bar{\phi}^{2}\right) .
$$

A diagonalizing linear transformation

yields

$$
z=\left(\begin{array}{l}
x \\
y
\end{array}\right)=\left(\begin{array}{cc}
1 & \gamma^{-1} \\
0 & 1
\end{array}\right)\left(\begin{array}{l}
\bar{\phi} \\
\bar{\psi}
\end{array}\right)
$$

$$
\dot{z}=\left(\begin{array}{cc}
0 & 0 \\
0 & -\gamma
\end{array}\right) z+\left(\begin{array}{c}
\gamma^{-1} \\
1
\end{array}\right)\left(x^{2}+\gamma^{-2} y^{2}-2 \gamma^{-1} x y\right)+\left(\begin{array}{c}
\gamma^{-1} \\
1
\end{array}\right)\left(b(t)-a_{\mathrm{sn}}\right)+o\left(x^{2}+y^{2}\right) .
$$

Now, using a quadratic transformation

$$
z=w+h(w), \quad w=\left(\begin{array}{l}
u \\
v
\end{array}\right)
$$

where $h(w)$ is a vector whose components are homogeneous quadratic polynomials in $(u, v)$, we kill the non-resonant quadratic terms in the last system, obtaining (cf. [2])

$$
\begin{aligned}
\left(\begin{array}{l}
u \\
v
\end{array}\right) & =\left(\begin{array}{cc}
0 & 0 \\
0 & -\gamma
\end{array}\right)\left(\begin{array}{l}
u \\
v
\end{array}\right)+\left(\begin{array}{l}
c_{1} u^{2} \\
c_{2} u v
\end{array}\right)+\left(I-h^{\prime}\right) Q+o\left(u^{2}+v^{2}\right), \\
Q & =\left(\begin{array}{c}
\gamma^{-1} \\
1
\end{array}\right)\left(b(t)-a_{\mathrm{sn}}\right),
\end{aligned}
$$

with $c_{1}, c_{2}>0$ independent of $k$.

We conclude from this the existence of a constant $\beta^{\prime}>0$ independent of $k$ such that in the $\beta^{\prime}$-neighbourhood of the origin in the $w$-plane the inequalities hold:

$$
\dot{u} \leq 2 c_{1} u^{2}+c k, \quad-2 \gamma v-c k \leq \dot{v} \leq-(\gamma / 2) v+c k \text {. }
$$

The image of the $\beta^{\prime}$-neighbourhood in the $w$-plane covers a $\beta$-neighbourhood of the saddle-node in the $(\phi, \dot{\phi})$-plane, where $\beta=\beta\left(\beta^{\prime}\right)$ is independent of $k$.

By part $1, \Phi\left(t_{\mathrm{sn}}\right)$ lies below $W^{s}$, and thus $u\left(t_{\mathrm{sn}}\right) \leq 0$. An explicit calculation using the estimates above shows that for some $c^{\prime}=c^{\prime}\left(\beta^{\prime}\right)>0$

$$
u(t) \leq \beta^{\prime} \quad \text { and } \quad|v(t)| \leq c k \quad \text { for } t_{\mathrm{sn}} \leq t \leq t_{\mathrm{sn}}+c^{\prime} k^{-1 / 2} \text {; }
$$

this translates into the desired statement (3.8).

Proof of lemma $B$ is based on comparison of solutions of equation (3.7) with those of the autonomous system (3.7)'. Let $\Phi(t)=(\phi, \dot{\phi})$ and $\Psi(t, a)$ be two solutions of (3.7) and (3.7)' respectively, with the same initial data $\Phi(0)=\Psi(0, a)$ satisfying initial inequalities (3.10) and (3.11), and let $\bar{\Psi}(t, a)$ satisfy the same equation (3.7)' but with initial conditions (2.4). We will show that $\Phi(t)$ enters the $r$-neighbourhood of the distinguished sink fast enough, thus proving the main parts (iii), (iv) of the lemma, by comparing $\Phi(t)$ with $\Psi$ and $\Psi$ with $\bar{\Psi}$. By definition of $a_{m}, \bar{\psi}\left(t, a_{m}\right)$ lies on the stable manifold of the distinguished saddle $\left(S a\left(a_{m}, k\right), 0\right)$. The distance between the first crossings of $\bar{\Psi}$ and $\Psi$ with the line $\phi=S a\left(a_{m}, k\right)-1 \equiv \phi_{1}$, chosen close but not too close to the saddle, is $\leq e^{-c / k}, c=c(\gamma)$, expressing the very weak 
dependence on the initial conditions after a long time. One obtains this exponential estimate by applying the divergence theorem to the strip in the $(\phi, \dot{\phi})$-plane bounded by two curves $\Psi\left(t, a_{m}\right)$ and $\bar{\Psi}\left(t, a_{m}\right)$, and the lines $\phi=\phi_{1}$ on the right and $\phi=\phi_{0} \equiv$ $\max \left(\phi\left(0, a_{m}\right), \bar{\phi}\left(0, a_{m}\right)\right)$ on the left. The inequality follows from the facts that the divergence of the phase flow of (3.7)' is $-\gamma$ and that the distance $\phi_{1}-\phi_{0}$ is $O\left(k^{-1}\right)$.

On the other hand, comparison of $\Phi(t)$ with $\Psi\left(t, a_{m}\right)$ shows that the intersection of $\Phi(t)$ with $\phi=\phi_{1}$ is above that for $\Psi\left(t, a_{m}\right)$ by at least $c \delta(k)$, with some $c>0$ independent of $k$, as a consequence of the conditions $b(t) \geq a_{m}+\delta(k)$. Recalling that $\delta(k) \geq e^{-k^{-p}}, p<1$, we conclude that $\Phi(t)$ crosses $\phi-\phi_{1}$ at a distance $\geq c \delta(k)$ above the stable manifold $\bar{\Psi}\left(t, a_{m}\right)$. This proves that $\Phi(t)$ enters and leaves an $O\left(k^{0}\right)$-neighbourhood of the saddle $S a\left(a_{m}, k\right)$ in time $\leq c \ln (1 / \delta(k))$.

Similar arguments show that $\Phi(t)$ passes under the stable manifold $\bar{\Psi}\left(t, a_{m+1}\right)$ of the saddle $S a\left(a_{m+1}, k\right)$ of equation $(3.7)^{\prime}$ with $a=a_{m+1}$, and at a safe distance $\geq c \delta(k)$, so that it takes time $\leq c \ln (1 / \delta(k))$ to enter the $r$-neighbourhood of the sink.

Proof of lemma $\mathrm{C}$ is contained in the first part of the proof of lemma A.

3.5. Proof of the existence of stable caterpillars. Our aim in this section is to show that both maps $P_{i j}: \Sigma_{i} \mapsto \tau^{m} \Sigma_{j}$ are into; according to the above remarks, this would imply the existence of a caterpillar solution. More precisely, we will show

$$
\forall C_{+}, C_{-}: \quad C_{+}-3 \pi>C_{-}+3 \pi>0, \quad \exists k_{0}=k_{0}\left(C_{+}, C_{-}\right),
$$

such that if $(I, k)$ lies in the intersection of a caterpillar domain $\mathscr{D}_{p, q}$ in figure 2.4 with the triangle given by

$$
1+T_{\mathrm{c}}+C_{-} k<I<1+T_{\mathrm{c}}+C_{+} k, \quad k<k_{0},
$$

then the Poincaré maps $P_{i j}$ are into.

To that end, we observe that the above restrictions on $(I, k)$ imply the following inequalities, where $c_{ \pm}=C_{ \pm} \mp 3 \pi>0$ :

$$
\begin{gathered}
k\left[S\left(I+\frac{k \pi}{2} ; k\right)-c_{+}\right]<a_{\mathrm{sn}}(k)<k\left[S\left(I+\frac{k \pi}{2} ; k\right)-c_{-}\right], \quad c_{+}>c_{-}>0, \\
I+k\left[S\left(\frac{k \pi}{2}, k\right)-c_{+}\right]<a_{\mathrm{sn}}(k)<I+k\left[S\left(\frac{k \pi}{2} ; k\right)-c_{-}\right], \\
\qquad\left|a_{m}(k)-\left(I+\frac{k \pi}{2}\right)\right|>\alpha k \\
\left|a_{m}(k)-\frac{\pi k}{2}\right|>\alpha k, \quad \forall m \in \mathbf{Z} .
\end{gathered}
$$

Before showing how these inequalities imply that the maps $P_{i j}$ are into, we give their physical interpretation. Rewrite (1.1) in the form

$$
\begin{gathered}
\ddot{\phi}_{1}+\gamma \dot{\phi}_{1}+\sin \phi_{1}+k \phi_{1}=I+k \phi_{2}, \\
\ddot{\phi}_{2}+\gamma \dot{\phi}_{2}+\sin \phi_{2}+k \phi_{2}=k \phi_{1},
\end{gathered}
$$

reminiscent of the autonomous system (2.1). If we place $\phi_{2}=\pi / 2$, then we can expect $\phi_{1}$ to tend to the distinguished sink $s=S(I+k \pi / 2)$ (lemma $\left.\mathrm{B}\right)$ : inequalities 
(3.12) say that the right-hand side of (3.16b) crosses the critical value $a_{\mathrm{sn}}$ when $\phi_{1}$ is between $s-c_{+}$and $s-c_{-}$; this crossing destroys the equilibrium in which $\phi_{2}$ has rested (figure 2.1), and we should expect $\phi_{2}$ to start, but slowly (lemma $A$ ) so that $\phi_{1}$ has ample time to settle into a sink (lemma B). For lemma B to be applicable, i.e. for $\phi_{1}$ to settle into a sink fast enough, we need $I+k \pi / 2 \approx$ right-hand side of (3.16a) to avoid 'bad' values of $a_{m}(k)$; hence we require (3.14).

Inequalities (3.13) and (3.15) are interpreted analogously.

Let $\Phi(0) \in \Sigma_{1}$; proof of the existence of $t=t_{2}$, such that $\Phi\left(t_{2}\right) \in \tau^{p} \Sigma_{2}$, consists of the following three observations on the dynamics of $\Phi(t)$.

Step 1. Fix $d>0, r>0$. For $k$ small enough, and $(I, k)$ satisfying (3.12) and (3.14), $\phi_{2}(t)$ will reach $\pi / 2+d$ at some $t=t_{1}<\infty$ and it will do so before $\phi_{1}(t)$ reaches
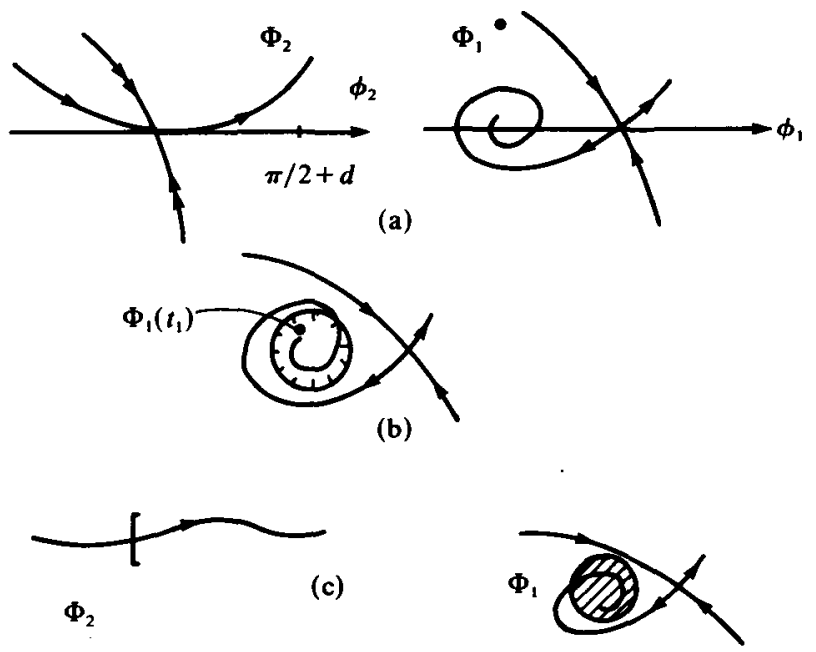

FIGURE 3.6. Illustration of the three steps in the proof of the existence of $t_{2}>0$ such that $\Phi\left(t_{2}\right) \in \Sigma_{2}$ if $\Phi(0) \in \Sigma_{1}$.

$S(I+k \pi / 2)+3 \pi$ (see figure $3.6(\mathrm{a}))$. That is, $\exists t_{1}$ such that

$$
\begin{gathered}
0 \leq \phi_{2}\left(t_{1}\right) \leq \pi / 2+d, \quad \phi_{2}\left(t_{1}\right)=\pi / 2+d, \\
\phi_{1}(t)<S(I+k \pi / 2)+3 \pi, \quad \forall t \leq t_{1} .
\end{gathered}
$$

Step 2. The time $t_{1}$ given by $\phi_{2}\left(t_{1}\right)=\pi / 2+d$ is large enough (if $k$ is small enough) for $\Phi_{1}(t)$ to enter an $r$-neighbourhood of $(S(I+k \pi / 2), 0)$ (see figure 3.6(b)); namely $t_{1} \geq c k^{-1 / 2}$ for some $c>0$ independent of $k$.

Step 3. $\Phi(t)$ crosses $\Sigma_{2}$ transversally at some $t=t_{2}>t_{1}$, i.e. $\Phi_{1}$ crosses the segment $S_{1}$ transversally while $\Phi_{2}$ lies in the box $B_{1}$ (figure 3.6(c)).

Proof of step 1. Assume the contrary, i.e. that $t_{1}=\infty$ or that $\phi_{1}$ reaches the indicated value first; while $0 \leq t \leq t_{1}$ we have $\phi_{2}(t)<\pi / 2+d$. The last inequality shows that $k \phi_{2}<k\left(\pi / 2+d_{1}\right)$ for $0 \leq t \leq t_{1}$ in equation (3.16a). Applying lemma $\mathrm{B}(\mathrm{v})$ to (3.16a), we deduce that $\phi_{1}\left(t_{1}\right)<S\left(I+k\left(\pi / 2+d_{1}\right)\right)+c$ (if $d_{1}, c$ are chosen $\left.<\pi / 2\right)<S(I+$ $k \pi / 2)+3 \pi$, which contradicts our assumption. 
Proof of step 2. Let us modify $\phi_{1}$ in equation (3.16b) by freezing it for $t \geq t_{1}$, i.e. define

$$
b(t)= \begin{cases}k \phi_{1}(t), & t \leq t_{1}, \\ k \phi_{1}\left(t_{1}\right), & t \geq t_{1} .\end{cases}
$$

With this modification of $\phi_{1}$ lemma $A$ becomes applicable to equation (3.16b) indeed, $b(t)$ satisfies (i)-(iii) (here we use step 1) and $\phi_{2}$ satisfies (iv) since $\Phi(0) \in \Sigma_{1}$. The lemma gives us: there exists $c>0$ such that

$$
\hat{\phi}_{2}(t)<\pi / 2+d \quad \text { for } 0<t<t_{\mathrm{sn}}+c k^{-1 / 2},
$$

where $\hat{\phi}_{2}$ is the solution of (3.16b) with $b(t)$ replacing the right-hand side $k \phi_{1}$, and $t_{\mathrm{sn}}$ is given by $b\left(t_{\mathrm{sn}}\right) \equiv k \phi_{1}\left(t_{\mathrm{sn}}\right)=a_{\mathrm{sn}}$.

From (3.19) and from the definition of $t_{1}: \hat{\phi}_{2}\left(t_{1}\right)=\phi_{2}\left(t_{1}\right)=\pi / 2+d$ it follows at once that $t_{1}>t_{\mathrm{sn}}+c k^{-1 / 2}$. With this bound on $t_{1}$ we turn our attention to (3.16a) to show that $\phi_{1}\left(t_{1}\right)$ belongs to an $r$-neighbourhood of $S(I+k \pi / 2)$. Indeed, equation (3.16a) for $\phi_{1}$ satisfies lemma $B$ for $0 \leq t \leq t_{1}$; the crucial point here is that the right-hand side $I+k \phi_{2}(t)$ avoids 'bad' values: for $0 \leq t \leq t_{1}$ we have

$$
\begin{aligned}
\left|b(t)-a_{m}\right| & =\left|I+k \phi_{2}-a_{m}\right|=\left|I_{k \pi / 2}-a_{m}+k\left(\phi_{2}-\pi / 2\right)\right| \\
& \geq\left|I+k \pi / 2-a_{m}\right|-k\left|\phi_{2}-\pi / 2\right| \\
& \geq(\alpha-d) k>(\alpha / 2) k
\end{aligned}
$$

if we pick $d<\alpha / 2$.

For $t \geq t_{1}$ these inequalities may fail; to make lemma $\mathrm{B}$ applicable, we set

$$
b(t)= \begin{cases}I+k \phi_{2}(t), & t \leq t_{1}, \\ I+k \phi_{2}\left(t_{1}\right), & t \geq t_{1} .\end{cases}
$$

Let $\hat{\phi}_{1}$ be the solution of (3.16a) with the right-hand side thus modified, with $\hat{\phi}_{1} \equiv \phi_{1}$ on $\left[0, t_{1}\right]$.

The remaining prerequisites of lemma $B$ hold for $\hat{\phi}_{1}$ (but not $\phi_{1}$ ) and we can conclude: $\exists 0<t^{\prime}<t^{\prime \prime}, t^{\prime \prime} \leq t^{\prime}+c \ln (1 / k)$ such that

$$
\begin{gathered}
\left|\tilde{\Phi}_{1}\left(t^{\prime \prime}\right)-(S(I+k \pi / 2), 0)\right|<r, \\
\tilde{\phi}_{1}\left(t^{\prime}\right)=S(I+k \pi / 2)-c_{+} .
\end{gathered}
$$

To complete step 2 , it remains to show that the capture (3.20) occurs early enough, i.e. $t^{\prime \prime}<t_{1}$, which would allow us to remove the tilde from (3.20), thus leading to the desired estimate.

To that end we note that

$$
k \phi_{1}\left(t_{\mathrm{sn}}\right)=a_{\mathrm{sn}}>k\left[S(I+k \pi / 2)-c_{+}\right]
$$

i.e.

$$
\phi_{1}\left(t_{\mathrm{sn}}\right)>S(I+k \pi / 2)-c_{+},
$$

implying the existence of $\bar{t}<t_{\mathrm{sn}} \dagger$ for which

$$
\phi_{1}(t)=S(I+k \pi / 2)-c_{+} .
$$

† This is the crucial point where we use (3.12); it is of essence that $t^{\prime}$ occurs before $k \phi_{1}=a_{\mathrm{sn}}$, i.e. before $\phi_{2}$ may start. 
This relation also holds for $\tilde{\phi}_{1}$ (since $\tilde{\phi}_{1} \equiv \phi_{1}$ on $\left[0, t_{1}\right]$ ); comparison with $(3.21)$ shows that $\bar{t}=t^{\prime}$ by the uniqueness of $t^{\prime}$ (lemma $\mathrm{B}(\mathrm{ii})$; choose $c_{6}=c_{+}>2 \pi$ ).

This shows that $t^{\prime}<t_{\mathrm{sn}}$ and thus

$$
t^{\prime \prime}<t^{\prime}+c \ln (1 / k)<t_{\mathrm{sn}}+c \ln (1 / k)<t_{\mathrm{sn}}+c k^{-1 / 2}<t_{1},
$$

with which $(3.20)$ becomes valid without the tilde:

$$
\left|\Phi_{1}\left(t_{1}\right)-(S, 0)\right|<r \text {. }
$$

Proof of step 3. The idea of the proof is simple: $\Phi_{1}$ is now (i.e. for $t \geq t_{1}$ ) trapped in the basin of the moving sink, while $\Phi_{2}(t)$ is running and thus will have to cross the line $\phi_{2}=\phi_{2}^{0}$ (at the proper height), provided $\phi_{2}^{0}$ is chosen large enough so that $\phi_{2}$ has enough time to settle into a running mode, and yet not too large for $\phi_{2}$ to stop before reaching $\phi_{2}^{0}$ due to the settling down of the running mode (see the heuristic argument). Here is the precise proof.

Consider two statements:

$$
\begin{gathered}
P\left(t_{1}, T\right)=\left[\frac{\pi}{2}+d \leq \phi_{2}(t)<\phi_{2}^{0}+2 \pi p \text { for all } t_{1} \leq t \leq T\right], \quad p=\left[\frac{S(I+k \pi / 2)}{2 \pi}\right], \\
Q\left(t_{1}, T\right)=\left[\left|\Phi_{1}(t)-S\left(I+k \phi_{2}(t)\right)\right|<r \text { for all } t_{1} \leq t \leq T\right]
\end{gathered}
$$

We have, using lemma $A$,

$$
P\left(t_{1}, T\right) \text { implies } Q\left(t_{1}, T\right)
$$

due to the choice of $\phi_{2}^{0}$ (given in the definition of $\Sigma_{2}$ ).

There exists $t_{2}>t_{1}$ for which $P$ fails but $Q$ still holds: $t_{2}=\sup \left\{T: P\left(t_{1}, T\right)\right.$ holds true\}. Clearly $t_{2}<\infty$, otherwise $Q\left(t_{1}, \infty\right)$ holds, and thus $\Phi_{1}$ forever lies in an $O\left(k^{0}\right)$-neighbourhood of $(S(I+k \pi / 2), 0)$ so that $\phi_{2}$ satisfies

$$
L \phi_{2}=k \phi_{1}=k\left[S(I+k \pi / 2)+O\left(k^{0}\right)\right]
$$

By lemma B $\phi_{2}$ must reach the value

$$
S\left(k\left[S(I+k \pi / 2)+O\left(k^{0}\right)\right]\right)-2 \pi>\phi^{0}+2 \pi p,
$$

which contradicts $P\left(t_{1}, \infty\right)$.

Summarizing, we have obtained $t_{2}>t_{1}$ such that

$$
\phi_{2}\left(t_{2}\right)=\phi_{2}^{0}+2 \pi p, \quad\left|\Phi_{1}\left(t_{2}\right)-S\left(I+k \phi_{2}\left(t_{2}\right)\right)\right|<r .
$$

By lemma $B(i)$ we have

$$
\frac{1}{2} p\left(\phi_{2}^{0}-2 \pi p, I+k \phi_{2}\left(t_{2}\right)\right)<\dot{\phi}_{2}\left(t_{2}\right)<2 p\left(\phi_{2}^{0}+2 \pi p, I+k \phi_{2}\left(t_{2}\right)\right) .
$$

This shows: $P_{12} \Phi(0) \equiv \Phi\left(t_{2}\right) \in \tau^{p} \Sigma_{2}$.

The proof of the into character of $P_{21}: \Sigma_{2} \rightarrow \tau^{-q} \Sigma_{1}$ goes similarly and is omitted.

3.6. Proof of the existence of the unstable caterpillar. We can represent $\sigma_{1}=S_{1} \times b_{1}$ as parallelepiped (see figure 3.7) in the three-dimensional hyperspace $\left\{\left(\phi_{1}^{0}, \psi_{1}, \phi_{2}, \psi_{2}\right)\right\}$ in $\mathbf{R}^{4}$, which we identify with $\mathbf{R}^{3}=\left\{\left(\psi_{1}, \phi_{2}, \psi_{2}\right)\right\}$ by dropping the first coordinate. Here we will show that the image of $\sigma_{1}$ in the section $\left(\phi_{2}=\phi_{2}^{0}+\right.$ $2 \pi p\} \supset \tau^{p} \sigma_{2}$ under Poincare map cuts across the parallelepiped $\sigma_{2}$ as shown in figure 3.2. Here $p$ is the same integer as in the previous section. 

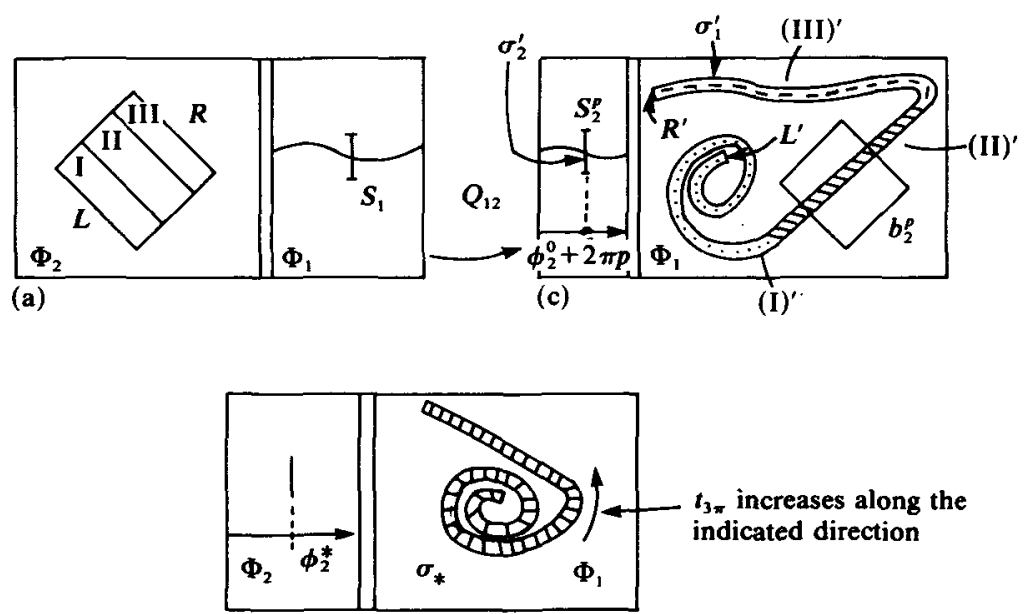

(b)

FIGURE 3.7. Images of $\sigma_{1}$ in the hyperplane $\phi_{2}=\phi_{2}^{0}+2 \pi p$ and the intermediate hyperplane $\left\{\phi_{2}=\phi_{2}^{*}\right\}$.

Superscripts $p$ (as in $b_{2}^{p}$ ) denote the $2 \pi p$-translates in the horizontal (i.e. $\phi_{1}$ or $\phi_{2}$ ) direction.

The precise meaning of this 'cutting across' is expressed in the following:

Theorem. If $(I, k) \in C_{p, q}$, where $C_{p, q}$ is an admissible domain, then the image $\sigma^{\prime}$ of the section $\sigma_{1}$ in the hyperplane $\left\{\phi_{2}=\phi_{2}^{0}+2 \pi p\right\}\left(\supset \tau^{p} \sigma_{2}\right)$ looks as shown in figure 3.2; its projections $\sigma_{1}^{\prime}$ and $\sigma_{2}^{\prime}$ onto $\sigma_{1}-$ and $\sigma_{2}$-planes are shown in figure $3.7(\mathrm{c})$.

More precisely, the box $b_{1}$ consists of three domains I, II, III such that (1)-(4) hold (see figure 3.7):

(1) (I)' $\cup(\mathrm{II})^{\prime}$ belongs to an $O(k)$-neighbourhood of the unstable manifold of the distinguished saddle of equation $L \phi=I+k \phi_{2}^{0}$. Here ( )' denotes the projection onto the $\Phi_{1}$-plane of the image under the Poincaré map $Q_{12}:(I)^{\prime}=\operatorname{proj}_{\Phi_{1}}\left(Q_{12}\left(b_{1} \times S_{1}\right)\right)$; (II)', (III)' are defined analogously.

(2) The image $L^{\prime}$ of the left boundary $L$ of $b_{1}$ lies to the left of $b_{2}^{p}$ (superscript $p$ denotes $2 \pi p$-translate in the horizontal (i.e. $\phi_{1}$ or $\phi_{2}$ ) direction). The image of the boundary II $\cap$ III lies to the right of $b_{2}^{p}$ (see figure 3.7).

(3) (III)' $\cap b_{2}=\varnothing$.

(4) Moreover, similar statements hold for the map $Q_{21}: \sigma_{2} \rightarrow\left\{\phi_{1}=\phi_{1}^{0}-2 \pi q\right\}$.

From (1)-(4) we conclude that the index of the map $\tau^{q-p} Q_{21}{ }^{\circ} Q_{12}: \sigma_{1} \rightarrow$ $\left\{\phi_{1}=\phi_{2}^{0}\right\} \supset \sigma_{1}$ is -1 , implying the existence of a fixed point. The corresponding running periodic solution is easily seen to be $a(p, q)$-caterpillar.

We outline the proof of the above theorem. The first step is to look at the section $\phi_{2}=\phi_{2}^{*}$, where $\phi_{2}^{*}=$ const $>\pi / 2$ is to be chosen shortly, i.e. to analyse the set of $\Phi_{1}$ when $\phi_{2}=\phi_{2}^{*}$. The reason for choosing such a section is this: as long as $\phi_{2} \leq \phi_{2}^{*}$, we have the bound $I+k \phi_{2} \leq I+k \phi_{2}^{*}$ in equation $L \phi_{1}=I+k \phi_{2} \equiv b$; this bound on the right-hand side makes it easy to estimate the set $\sigma_{*}=\left\{\Phi_{1}\left(t_{2}\right): t_{2}=t_{2}(\Phi(0))\right.$ defined by $\left.\phi_{2}\left(t_{2}\right)=\phi_{2}^{*}\right\}$. 
This set $\sigma_{*}$ is sketched in figure 3.7(b). It is easy to prove that, given any $C>0$, there exists $k_{0}=k_{0}(C)$ such that $\forall k<k(C)$ there exists a partition $\sigma_{*}=\mathrm{I}_{*} \cup \mathrm{II}_{*} \cup$ $\mathrm{II}_{*}$, where $\mathrm{I}_{*} \subset O(k)$-neighbourhood of $W^{\mathrm{u}}$ (the unstable manifold of the distinguished saddle for $L \phi=I), I_{*} \subset O(k)$-neighbourhood of the upper branch of $W^{s}$, and that $\sup \left\{\phi_{1}\right.$-projection of $\left.\mathrm{III}_{*}\right\}=S a(I)-C_{*}$. Pre-images of $\mathrm{I}_{*}$, etc. in $\sigma_{1}$ define I, etc. of the above theorem.

The above statement uses only the bound on $b(t)$ and the fact that $\Phi(0) \in S_{1}$; it is a statement on the non-autonomous one-degree-of-freedom systems $L \phi=b$.

Proof of (1). The first assertion of the theorem is proven as follows: (i) the unstable manifold of the saddle of $L \phi_{1}=I+k \phi_{2} \equiv b$ nearest $S a(I)$ varies with the speed $O(k)$; (ii) with any $b$ frozen between $I+k \phi_{2}^{*}$ and $I+k\left(\phi_{2}^{0}+2 \pi p\right)$ (i.e. $\phi_{2}^{*}<\phi_{2}<\phi_{2}^{0}+$ $2 \pi p)$, this manifold is an attracting set with the exponential rate of attraction $O\left(k^{0}\right)$, uniformly in $\phi_{1}$. Thus any point in II $\cup$ III will enter (and will stay in) the $O(k)$ neighbourhood of the unstable manifold before $\phi_{2}$ will reach $\phi_{2}^{0}+2 \pi p=O(1 / k)$. Proof of (2) makes crucial use of the inclusion $(I, k) \in C_{p, q}$. The fact that $L^{\prime}$ is near the sink and thus lies to the left of $b_{2}^{p}$ follows from the arguments of the previous section: these solutions are close to the stable caterpillar. The key step is to trace the evolution of the boundary $\partial_{*} \equiv \mathrm{II}_{*} \cap \mathrm{III}_{*}$ in order to show that $\partial^{\prime}$ lies to the right of $b_{2}^{p}$.

To that end it suffices to show that $\phi_{1}$ reaches the value $S a(I)+2 \pi$ before $\phi_{2}$ reaches $\phi_{2}^{0}$. Here $\left(\phi_{1}, \dot{\phi}_{1}, \phi_{2}, \dot{\phi}_{2}\right)$ starts in our intermediate section $\partial_{*} \times\left\{\phi_{2}=\phi_{2}^{*}\right\}$ at $t=t^{*}$. We have $L \phi_{1}=I+k \phi_{2} \geq I+k \phi_{2}^{*}=$ const for $t \geq t^{*}$, and, moreover, $\Phi_{1}\left(t^{*}\right) \epsilon$ $\partial_{*}$ lies above the solution of $L x=I$ with $X(0)=(x, \dot{x})_{t=0} \in S_{1}$ (see figure 3.8).

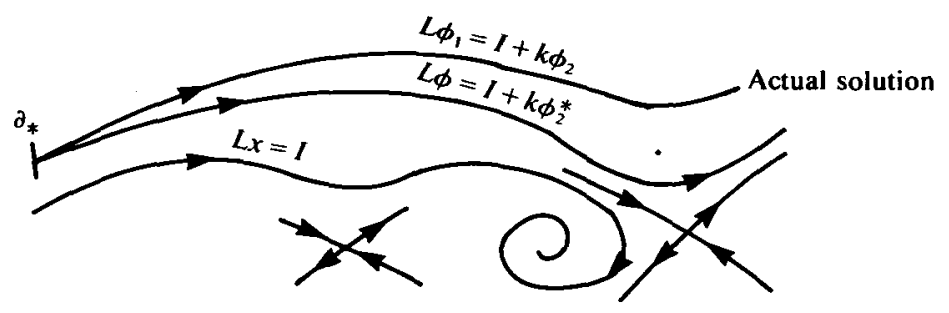

FigurE 3.8. Showing that $\phi_{1}$ reaches $S a(I ; k)+2 \pi$ before $\phi_{2}$ reaches $\phi_{2}^{0}$.

This allows us to compare $\phi_{1}$ subject to $L \phi_{1} \geq I+k \phi_{2}^{*}$ with $\phi$ subject to $L \phi=I$. It is not hard to prove that if $\phi_{2}^{*}$ is chosen sufficiently large, then $\phi_{1}(t)$ will go beyond $S a(I)+2 \pi$ in time $T \leq c \ln (1 / k)$. Moreover, $\phi_{2}^{*}$ can be chosen independent of $k$, and thus the time it takes for $\phi_{2}$ to go from $\phi_{2}^{*}$ to $\phi_{2}^{0}$ is $O\left(k^{-1}\right)>c \ln (1 / k)=T$. That is, $\phi_{1}$ reaches $S a(I)+2 \pi$ first. This proves (2).

Proof of (3) uses the facts that all solutions starting in $\mathrm{III}_{*}$ satisfy differential inequality

$$
L \phi_{1}=I+k \phi_{2} \geq I+k \phi_{2}^{*},
$$

and that $\phi_{1}(0) \in I I I_{*}$ lie above the solution $X(t)$ of $L x=I$ with $X(0) \in S_{1}$. The argument is the same as in the proof of (2). 
3.7. Proof of stability. In this section we will show that the caterpillar solutions found in $\S 3.5$ are indeed stable.

Let $\Phi(t)$ be the caterpillar solution found in $\S 3.4$. We will construct five sections $\Sigma^{i}, i=1, \ldots, 5$ (figure 3.9) transversal to $\Phi(t), \Sigma^{5}$ being a $\tau$-translate of $\Sigma^{1}$, such that the composition $M=M^{4} \circ M^{3} \circ M^{2} \circ M^{1}: \Sigma^{1} \rightarrow \Sigma^{5} \equiv \Sigma^{1} \bmod \tau$ of Poincaré maps $M^{i}: \Sigma^{i} \rightarrow \Sigma^{i+1}$ is a contraction. Such decomposition of $M$ corresponds to two running stages and to two transitional stages during one period. The running stages give rise to an exponentially strong contraction, while the transitional stages cannot overcome this effect.

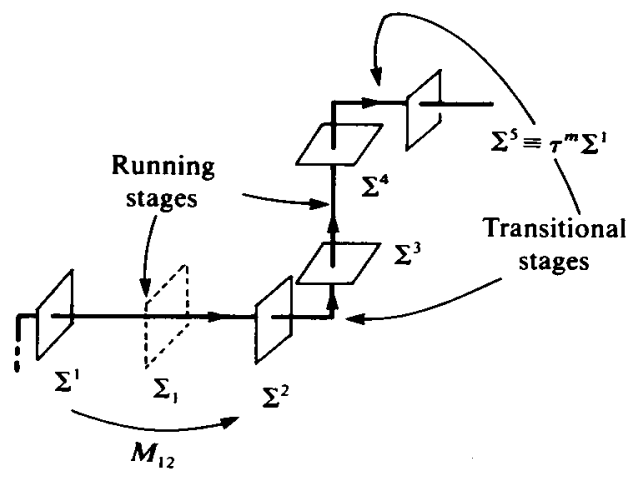

Figure 3.9. Decomposition of the Poincaré map $M: \Sigma^{1} \rightarrow \Sigma^{5} \equiv \tau^{p-q} \Sigma^{1}$.

We define

$$
\begin{aligned}
& \Sigma^{1}=\left\{\Phi: \phi_{1}=2 \pi(q+1 / \sqrt{k})\right\}, \\
& \Sigma^{2}=\left\{\Phi: \phi_{1}=2 \pi(p-1 / \sqrt{k})\right\}, \\
& \Sigma^{3}=\left\{\Phi: \phi_{2}=2 \pi(1 / \sqrt{k})\right\}, \\
& \Sigma^{4}=\left\{\Phi: \phi_{2}=2 \pi(p-q-1 / \sqrt{k})\right\}, \\
& \Sigma^{5}=\left\{\Phi: \phi_{1}=2 \pi(p+1 / \sqrt{k})\right\} .
\end{aligned}
$$

Let $0=t^{1}<t^{2}<t^{3}<t^{4}<t^{5}=T$ be the times when $\Phi(t)$ crosses $\Sigma^{i}$.

Sections $\Sigma^{1}, \Sigma^{2}$ have natural coordinates $\left(\psi_{1}, \phi_{2}, \psi_{2}\right)$, while $\Sigma^{3}, \Sigma^{4}$ are described by $\left(\phi_{1}, \psi_{1}, \psi_{2}\right)$.

Since $\Phi(t)$ is transversal to every $\Sigma^{i}$, the Poincaré maps $M_{i}: \Sigma^{i} \rightarrow \Sigma^{i+1}, i=1,2,3,4$ are well defined near $\Phi\left(t^{i}\right)$. The proof of stability reduces to showing that there exist constants $c_{1}, c_{2}>0$ such that (for $k>0$ small enough) we have

$$
\left|d M_{i}\left(\Phi\left(t^{i}\right)\right)\right| \leq e^{-c_{i} / k}, \quad i=1,3,
$$

and

$$
\left|d M_{i}\left(\Phi\left(t^{i}\right)\right)\right| \leq e^{c_{2} / \sqrt{ } k}, \quad i=2,4 .
$$

Here $|\cdot|$ can be (and is) chosen as the Euclidean norm in $\Sigma^{i}$. Indeed, these estimates yield at once

$$
|d M| \leq e^{-2 c_{1} / k+2 c_{2} / \sqrt{ } k}<1
$$

for $k$ small enough, showing that $\Phi(t)$ is exponentially stable. 
Proof of (3.24). Estimate (3.24) for $i=1$ ( $i=3$ is analogous) follows from the fact that for $t^{1} \leq t \leq t^{2}$ we have, roughly speaking, a direct product of contractions, one in the $\Phi_{2}$-plane, the other in the $\Phi_{1}$-direction, as is clear from figure 3.10.

The rate of these contractions is $O\left(k^{0}\right)$ during the time interval $\left[t^{1}, t^{2}\right]$ of length $O\left(k^{-1}\right)$, leading to (3.24). We omit further details.

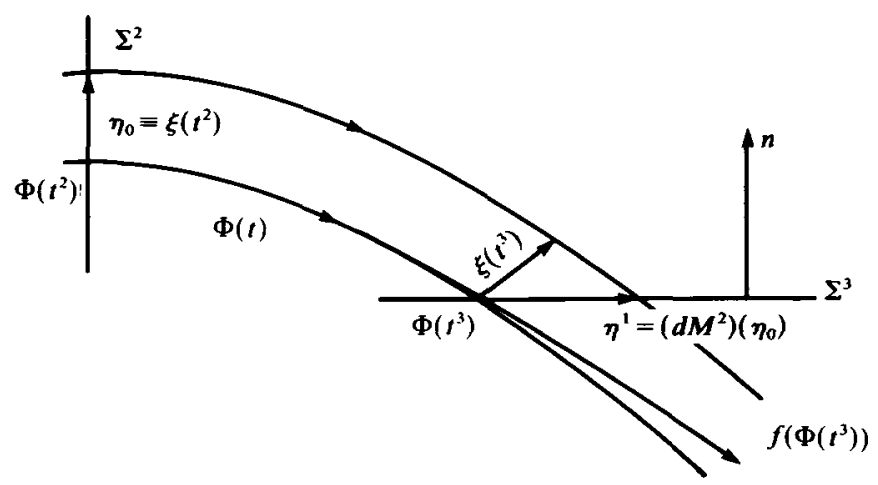

FIGURE 3.10. Derivative of the Poincaré maps in terms of solutions $\xi(t)$ of the linearized equation.

Proof of (3.25) for $i=2$ ( $i=4$ is analogous) rests on the observation that the linear map $d M_{2}: T_{\Phi\left(t^{2}\right)} \Sigma^{2} \rightarrow T_{\Phi\left(t^{3}\right)} \Sigma^{3}$ is related to the linearization of (2.2) around $\Phi(t)$ as follows:

$$
\left(d M_{2}\right) \eta_{0} \equiv \eta_{1}=\xi\left(t^{3}\right)-\frac{\left(\xi\left(t^{3}\right), n\right)}{\left(f\left(\Phi\left(t^{3}\right), n\right)\right)} f\left(\Phi\left(t^{3}\right)\right)
$$

(see figure 3.10), where $\xi=\xi(t)$ is the solution of the linearization of (2.2) around $\Phi(t)$, with $\xi\left(t^{2}\right) \equiv \eta_{0}, n$ is a unit vector normal to $\Sigma^{3}, f(x)$ is the vector field (2.2), and $($, ) denotes the Euclidean inner product.

Now, since $t^{3}-t^{2} \leq c / \sqrt{k}$, we have

$$
\left|\xi\left(t^{3}\right)\right| \leq\left|\xi\left(t^{2}\right)\right| \exp \left[\left(t^{3}-t^{2}\right) \sup |d f(\Phi(t))|\right] \leq\left|\eta_{0}\right| \exp (c / \sqrt{k}),
$$

which together with (3.26) gives

$$
\left|\left(d M_{2}\right) \eta_{0}\right| \leq e^{c / \sqrt{ } k}\left|\eta_{0}\right|
$$

thus proving (3.25). Here we have made a crucial use of the fact that the flow $f$ is 'sufficiently transversal' to $\Sigma^{3}$, i.e. that the denominator in (3.26) is bounded from below.

Acknowledgements. Supported in part by the NSF and DARPA. Part of this work was carried out by the author at MSRI, Berkeley.

\section{REFERENCES}

[1] A. A. Andronov, E. A. Vitt \& S. E. Khaikin. Theory of Oscillations. Princeton University Press, Princeton, NJ (1949). 
[2] V. I. Arnold. Geometrical Methods in the Theory of Ordinary Differential Equations. Springer, New York (1983).

[3] D. K. Campbell, M. Peyrard \& P. Sodano. Kink-antikink interactions in the double sine-Gordon equation. Physica 19D (1986), 165-205.

[4] L. Chua, Y. Yao \& Q. Yang. The devil's staircase in circuits. Int. J. Circuit Th. Appl. (1986).

[5] M. Cirillo, R. D. Parmentier \& B. Savo. Mechanical analog studies of a perturbed sine-Gordon equation. Physica 3D (1981), 565-576.

[6] G. Grüner \& A. Zettl. CDW conduction: a novel collective transport phenomenon in solids. Phys. Rep. 119 (1985), 119-232.

[7] Y. Imry \& L. Schulman. Qualitative theory of the nonlinear behavior of coupled Josephson junctions. J. Appl. Phys. 49 (1978), 749-758.

[8] B. D. Josephson. Phys. Lett. 1 (1962), 251.

[9] D. N. Landberg, D. J. Scalapino \& B. N. Taylor. The Josephson effects. Sci. Amer. 21, 30-39.

[10] M. Levi. Beating modes in the Josephson junction. In Chaos in Nonlinear Dynamical Systems, ed. J. Chandra. SIAM, Philadelphia (1984), 56-73.

[11] M. Levi, F. C. Hoppensteadt \& W. L. Miranker. Dynamics of the Josephson junction. Q. Appl. Math. (1978), 167-198.

[12] P. M. Marcus \& Y. Imry. Steady oscillatory states of a finite Josephson junction. Solid State Commun. 33 (1980), 345-349.

[13] P. M. Marcus, Y. Imry \& E. Ben-Jacob. Characteristic modes and the transition to chaos of a resonant Josephson circuit. Solid State Commun. 41 (1982), 161-166.

[14] J. Matisoo. Josephson-type superconductive tunnel junctions and applications. IEEE Trans. Magn. 5 (1969), 848-873.

[15] R. F. Miracky, M. H. Devoret \& J. Clarke. Deterministic hopping in a Josephson circuit described by a one-dimensional mapping. Phys. Rev. A 31 (1985), 2509-2519.

[16] Nakajima, K. T. Yamashita \& Y. Onodera. Mechanical analogue of active Josephson transmission line. J. Appl. Phys. 45 (1974), 3141-3145.

[17] F. Odeh. On existence, uniqueness and stability of solutions of the Josephson phase equation. Preprint.

[18] A. C. Scott. A nonlinear Klein-Gordon equation. Amer. J. Phys. 37 (1969), 52-61.

[19] M. P. Soerensen, N. Arley, P. L. Christiansen, R. D. Parmentier \& O. Skovgaard. Intermittent switching between soliton dynamic states in a perturbed sine-Gordon model. Phys. Rev. Lett. (1983), 1919-1922.

[20] J. J. Stoker. Nonlinear Vibrations. Interscience Publishers, New York (1950).

[21] D. B. Sullivan \& J. E. Zimmerman. Mechanical analogs of time dependent Josephson phenomena. Amer. J. Phys. 39 (1971), 1504-1517.

[22] F. Tricomi. Integrazione di un'equazione differenzale presentasi in elettrotecnica. Ann. Scuolo Norm. Sup. Pisa 2 (1933).

[23] M. St. Vincent. Preprint.

[24] J. E. Zimmerman \& D. B. Sullivan. High-frequency limitations of the double-junction SQUID amplifier. Appl. Phys. Lett. 31 (1977), 360-362. 